\title{
Colaboração para a Reparação de Alinhamento de Ontologias
}

\author{
Miriam Oliveira dos Santos ${ }^{1}$, Tadeu Moreira de Classe $^{1}$, Carlos Eduardo Mello ${ }^{1}$ \\ ${ }^{1}$ Programa de Pós-Graduação em Informática (PPGI) \\ Departamento de Informática Aplicada (DIA) \\ Universidade Federal do Estado do Rio de Janeiro (UNIRIO) \\ Rio de Janeiro - RJ - Brasil \\ \{miriam.santos, tadeu.classe, mello\}@uniriotec.br
}

\begin{abstract}
The process of ontology aligning repair consists of ratifying and confirm the alignment of elements among two or more ontologies. Collaborative resources, when applied to the process of ontology alignment repair, can help the domain experts in that task once they should share knowledge among them, improving the ontology alignment result. Thus, this work aims to present and analyze RAOSystem's collaborative resources, which were developed based on the $3 C$ collaboration model. The study validation was made using a quasiexperimental study with domain experts. During the study execution, we collected a system $\log$ and a survey. As result, the collaborative resources as comments, acceptance, and rejection were the most used. And some participants collaborate more them others. In this sense, we discovered clues that collaborative resources contributed to the domain experts task, however new studies about the impacts and implications of collaborative work in this context need to be performed.
\end{abstract}

Resumo. O processo de reparação de ontologias consiste em corrigir elou confirmar alinhamentos entre elementos de duas ou mais ontologias. Recursos colaborativos quando aplicados ao processo de reparação de alinhamento de ontologias podem auxiliar os especialistas de domínio, já que eles devem compartilhar conhecimento entre si, melhorando o resultado deste alinhamento. Este trabalho objetiva verificar os recursos colaborativos do sistema RAOSystem, implementados com base no modelo de colaboração $3 C$. A análise foi feita em um quasi-experimento com especialistas de domínio. Durante a tarefa, um log de uso do sistema foi coletado, além de um questionário de opinião ao final do estudo. Como resultado, os recursos como comentários, aceitação e rejeição de termos foram mais usados. E, aparentemente, alguns participantes colaboraram mais que outros. Assim, foi obtido indícios que os recursos colaborativos contribuíram com a tarefa dos especialistas, embora estudos futuros sobre impactos e implicações ainda precisem ser conduzidos.

\section{Introdução}

Pesquisas relacionadas ao campo da e-Science há algum tempo tem como base o compartilhamento de dados e métodos para a recuperação, integração e interoperabilidade de recursos científicos entre as comunidades científicas [Brodaric e Gahegan 2010, Meng et al. 2016]. Neste campo, as ontologias são aplicadas como componentes 
principais de sistemas que buscam a organização e compartilhamento de conhecimento relacionado a um domínio específico [Euzenat e Rousset 2020].

A bioinformática é uma área que é constantemente relacionada à e-Science devido a necessidade execução de experimentos, simulações computadorizadas e da troca de conhecimento entre grandes centros de pesquisa [Harrow et al. 2019]. Estes centros (ex.: European Molecular Biology Laboratory $(E M B L-E B I)^{1}$, Alliance of Genome Resources ${ }^{2}$ e Monarch Initiative ${ }^{3}$ ) visam a interoperabilidade de dados clínicos e pesquisas em diversas partes do mundo, buscando a colaboração, difusão de informação a partir de novas descobertas relacionadas a doenças e medicamentos, aplicando ontologias frente a problemas reais [Harrow et al. 2019, Banouar e Raghay 2016].

$\mathrm{Na}$ maioria das vezes é comum que existam diferentes ontologias para descrever um mesmo conhecimento sobre um domínio. Nestes casos, elas apenas são modeladas ou organizadas de formas distintas umas das outras. Por exemplo, é fácil se deparar com ontologias que organizam o conhecimento sobre doenças sendo modeladas de maneira distintas [Harrow et al. 2019].

Visando a interoperabilidade, e na tentativa de diminuir a distância entre as ontologias de um mesmo domínio, é feito um processo conhecido como alinhamento de ontologias [Gargouri e Jaziri 2010]. Este processo consiste em destacar relacionamentos e/ou correspondências entre os termos e conceitos pertencentes as ontologias, relacionando-os de forma a diminuir sua heterogeneidade semântica [Silva et al. 2016]. Como resultado, é gerado um mapeamento que contempla um conjunto de correspondências entre os conceitos de duas ou mais ontologias [Euzenat 2014].

O alinhamento entre ontologias pode ser realizado por métodos automáticos, semiautomáticos ou manuais [Meilicke 2011]. Os automáticos consistem na aplicação de estratégias como: comparação textual, linguística, alinhamento estrutural e semântico etc. O manual, consiste de os especialistas de domínio destacarem os termos correspondentes entre as ontologias [Euzenat et al. 2007]. E os semi-automáticos consideram a utilização de um método para propagar implicações de decisões tomadas na revisão dos mapeamentos com o objetivo de reduzir significativamente o esforço de revisão em termos de números de decisões que devem ser tomadas pelos especialistas [Meilicke et al. 2008, Meilicke et al. 2009].

Contudo, os métodos de alinhamentos automáticos não são totalmente efetivos e muitas vezes podem não relacionar termos de maneira correta [Li et al. 2019]. Devido à complexidade dos processos de alinhamentos automáticos é preciso que os especialistas de domínio realizem um trabalho de validação e reparação para garantir sua do mapeamento gerado [Xue e Liu 2017].

Diferentes especialistas de domínio possuem diferentes visões sobre um determinado contexto ou área. Essa combinação de conhecimento e a colaboração entre os indivíduos na realização de tarefas de reparação de alinhamento de ontologias (RAO), gera mais conhecimento útil com maior valor agregado que o conhecimento individual

\footnotetext{
${ }^{1}$ https://www.ebi.ac.uk/spot/oxo/search

${ }^{2}$ https://www.alliancegenome.org/

${ }^{3}$ https://monarchinitiative.org
} 
[Xue e Liu 2017]. Desta forma, sistemas colaborativos podem apoiar a tarefa de RAO, de modo a diminuir a carga de trabalho dos especialistas, aumentar o valor do conhecimento construído e garantir a geração de um mapeamento entre ontologias com maior qualidade [Borges 2011, Xue e Liu 2017].

Este trabalho objetiva apresentar e verificar o sistema RAOSystem, construído a partir do modelo MRED (Modelo de desenvolvimento de sistemas para Reparação de ontologias para Especialistas de Domínio) [Santos 2020, Santos et al. 2020], a respeito de seus recursos colaborativos. O sistema implementa requisitos e funcionalidades colaborativas, tendo como inspiração o modelo de colaboração 3C [Fuks et al. 2008], requisitos estes previstos no modelo MRED. Os requisitos colaborativos foram avaliados através de um estudo quasi-experimental, sendo os dados analisados através de abordagens qualitativas e quantitativas, visando verificar se os elementos colaborativos contribuíram com os especialistas de domínio na tarefa de RAO.

Assim, este artigo se organiza em: seção 2, com definições sucintas sobre os principais conceitos da pesquisa relacionados ao alinhamento e reparação de ontologias e o modelo de colaboração 3C. A seção 3 aborda alguns trabalhos relacionados. Na seção 4 é apresentado o RAOSystem, e como seus recursos colaborativos foram inspirados do modelo 3C. A seção 5 descreve o planejamento do estudo e a análise dos resultados. E, finalmente, a seção 6 apresenta uma conclusão e passos futuros da pesquisa.

\section{Bases Teóricas}

\subsection{Alinhamento e Reparação de Ontologias}

Para promover a interoperabilidade entre sistemas que usam as ontologias como fonte de conhecimento e informação é necessário realizar o alinhamento entre seus conceitos [Silva et al. 2016]. O alinhamentos de ontologias é um processos capaz de auxiliar no processo de mapeamento semântico entre duas ou mais ontologias, relacionando os conceitos do domínio [Gargouri e Jaziri 2010]. Como resultado deste processo, um mapeamento de ontologias é gerado. Esse mapeamento compreende um conjunto de correspondências que incluem a a representação do relacionamento pode ser de equivalência, disjunção ou associação e, um valor que representa a força de similaridade entre os termos [Banouar e Raghay 2016].

Existem diferentes técnicas para realização do alinhamento de ontologias, as quais se baseiam em comparação textual (string), linguística, estrutural e semântica etc [Banouar e Raghay 2016]. Mas, mesmo que haja diferentes técnicas automáticas para o alinhamento de ontologias, elas não são completamente efetivas [Li et al. 2019]. Devido a essa complexidade da tarefa de alinhamento, a intervenção humana do especialista de domínio é necessária para verificar e ajustar inconsistências no mapeamento, levando a resultados mais precisos [Li et al. 2015].

Devido a isso, é necessário uma tarefa subsequente, conhecida por Reparação de Alinhamento de Ontologias [Pesquita et al. 2013]. Essa tarefa consiste no refinamento do alinhamento, através da análise do especialista de domínio, o qual pode incluir, remover ou confirmar correspondências entre termos [Banouar e Raghay 2016]. A tarefa também permite a identificação de inconsistências lógicas, tornando o alinhamento final mais consistente [Pesquita et al. 2013]. A implementação de ações de RAO podem ser feitas 
usando estratégias automatizadas ou manuais [Meilicke 2011]. E no uso de estratégias manuais, práticas colaborativos devem ser aplicadas para que os especialistas de domínio consigam compartilhar, refletir e construir um conhecimento em conjunto, gerando mais qualidade ao mapeamento de ontologias [Falconer et al. 2007].

\subsection{O Modelo de Colaboração 3C}

O Modelo 3C de Colaboração [Fuks et al. 2008] analisa a colaboração sob a ótica de 3 dimensões: Comunicação, que possibilita a troca de mensagens, argumentação e negociação entre os envolvidos na colaboração; Coordenação, cuidando do gerenciamento e controle da colaboração, as pessoas envolvidas e como elas interagem, executam atividades e usam recursos no espaço compartilhado; e Cooperação, ou o trabalho que as pessoas desenvolvem em conjunto no espaço compartilhado. Esse modelo também destaca a Percepção, que surge da aplicação das outras dimensões, envolvendo mecanismos que permitem saber que participante da colaboração fez o "quê, quando e onde", além o impacto que uma atividade terá sobre o trabalho dos demais membros do grupo. Além desses elementos explícitos no modelo 3C, é importante destacar que a colaboração possibilita a troca de conhecimento pelo grupo (ou conhecimento coletivo), gerando a Memória Coletiva, a qual está disponível aos membros do grupo sempre que necessário [Fuks et al. 2008, Mistrík et al. 2010].

\section{Trabalhos Relacionados}

Existem iniciativas na literatura para a tarefa de RAO, porém elas não fornecem o suporte necessário para os especialistas de domínio, muito menos ao trabalho colaborativo [Santos 2020]. Essas ferramentas se limitam apenas em exibir os mapeamentos que elas mesmas conseguiram elencar. Dentre as principais delas é possível destacar: COMA (Combination of Schema Matching Approach), fornecendo uma plataforma para analisar mapeamentos, representando-os em árvores de conceitos [Massmann et al. 2011]. ASMOV (Automated Semantic Matching of Ontologies With Semantic Validation), apresentando uma página web contendo os termos da ontologia e seus respectivos relacionamentos [Meilicke 2011]. E, AML (Agreement Maker Light), que segundo [Faria et al. 2013], apresenta grafos como forma de representação das ontologias.

Contudo a colaboração na tarefa de alinhamento de ontologias, especificamente na validação do mapeamento gerado pelas ferramentas automáticas não é uma questão nova. Neste sentido, [Xue e Liu 2017] propôs o Compact Interative Memetic Algorithm (CIMA), baseado em sistemas de alinhamento de ontologia colaborativos a fim de reduzir a carga de trabalho dos especialistas de domínio envolvidos. No trabalho [Karampatakis et al. 2018] é proposto o sistema Alignment, um sistema colaborativo para o alinhamento de ontologias interativo, fornecendo um ambiente simples apoiado por algoritmos de similaridade. [Smirnov e Shilov 2013] propõem um sistema de recomendação colaborativo para o alinhamento de ontologias, baseando-se em características como o perfil de usuário, gestão de contexto, serviços web etc. Os autores consideram que esse sistema contribui para acelerar o trabalho de alinhamento.

Especificamente sobre a construção de sistemas colaborativos, sua complexidade de construção, sugiram frameworks e kits para facilitar a sus criação. Esses 
kits apresentam componentes especialidades, desenvolvidos especificamente para promover a colaboração. Como por exemplo, é possível citar o GroupKit [Roseman e Greenberg 1995], o qual fornece componentes como: rolagem, multiusuários, área compartilhada, chats etc.

O sistema RAOSystem, objeto de estudo deste artigo, se inspirou na literatura e na técnicas de trabalhos como os mencionados acima. Como o mesmo foi desenvolvido com base no modelo MRED, foi instanciado no sistema funcionalidades de visualização interativa e colaborativas entre os especialistas de domínio, além da implementação de recursos como os componentes descritos pelo GroupKit, por exemplo.

\section{Reparação de Alinhamentos de Ontologias de Forma Colaborativa}

O RAOSystem é um sistema de informação com o propósito de dar suporte aos especialistas de domínio na tarefa de reparação de alinhamento de ontologias [Santos et al. 2020]. Sua implementação foi guiada a partir das especificações encontradas em cada uma das dimensões previstas no modelo MRED, sendo: compreensão de ontologias (CO), visualização de informações (VI), tempo gasto (TG), reparação de alinhamento (RA) e, principalmente o trabalho colaborativo (TC). A dimensão de trabalho colaborativo foi construída a partir do Modelo de Colaboração 3C [Fuks et al. 2008], o qual inspirou as principais recomendações do MRED sob esta dimensão e, consequentemente, as funcionalidades colaborativas implementadas no RAOSystem. Neste sentido, na Figura 1 é possível contemplar as principais funcionalidades inseridas no sistemas com base no Modelo 3C.

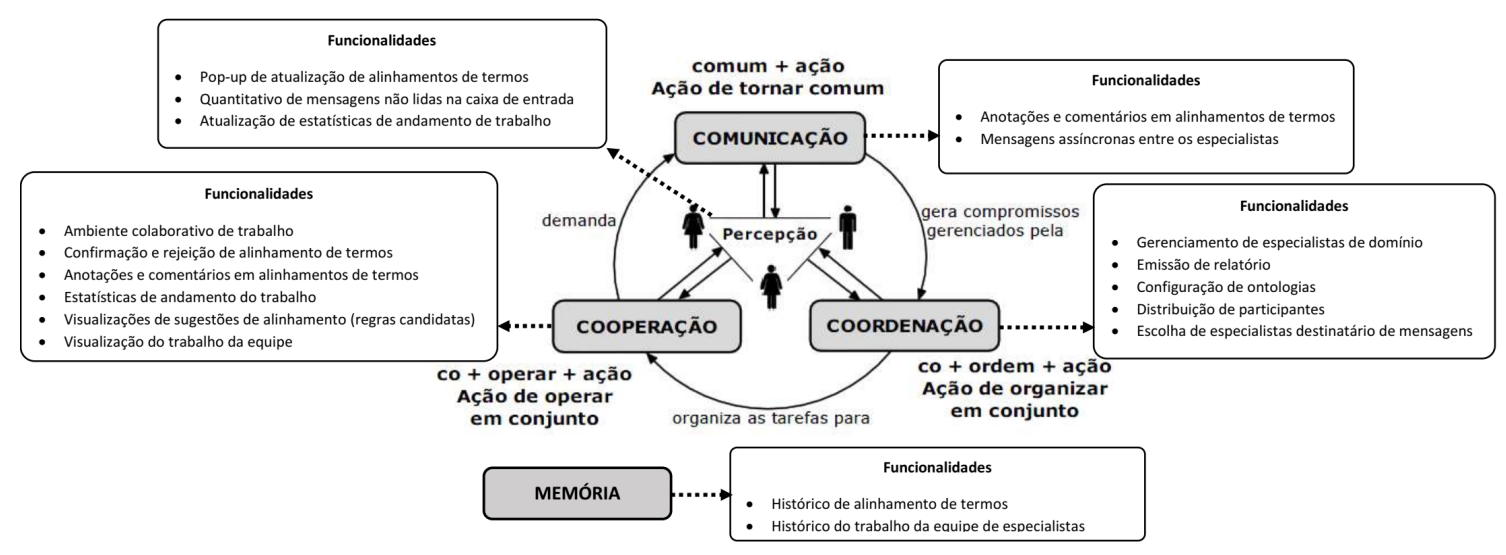

Figura: 1. Modelo $3 \mathrm{C}$ com as funcionalidades colaborativas do RAOSystem (Adaptado de [Pimentel e Fuks 2012])

Considerando a dimensão de Comunicação o RAOSystem, duas principais funcionalidades foram incluídas: mensagens assíncronas (Figura 2A) para a comunicação de um ou mais especialistas de domínio e o comentários em alinhamento de termos (Figura 2B), no qual é possível tirar dúvidas e dar sugestões sobre o alinhamento. A possibilidade de comentar os alinhamentos também pode ser considerado parte da dimensão de Cooperação, a qual também envolve o ambiente de trabalho colaborativo, a confirmação e rejeição de alinhamentos (Figura 3A), observação do trabalho da equipe (Figura 3B), visualização de ontologias, organização de grupos de similaridade entre os termos (Figura 3C), estatísticas de andamento do trabalho (Figura 5A) etc. Em 
relação a Coordenação, existe a possibilidade de gerenciar os especialistas de domínio e ontologias (Figura 4B), emissão de relatórios e a distribuição do trabalho (Figura 4A). Os mecanismos de Percepção se apoiam em pop-ups (Figura 5B) de notificação de alterações no alinhamento feito por outros especialistas e nas estatísticas de andamento do trabalho (Figura 5A), ambos em tempo real. E finalmente a Memória, disponibilizando o histórico individual (Figura 6A), quanto do trabalho em grupo (Figura 6B).

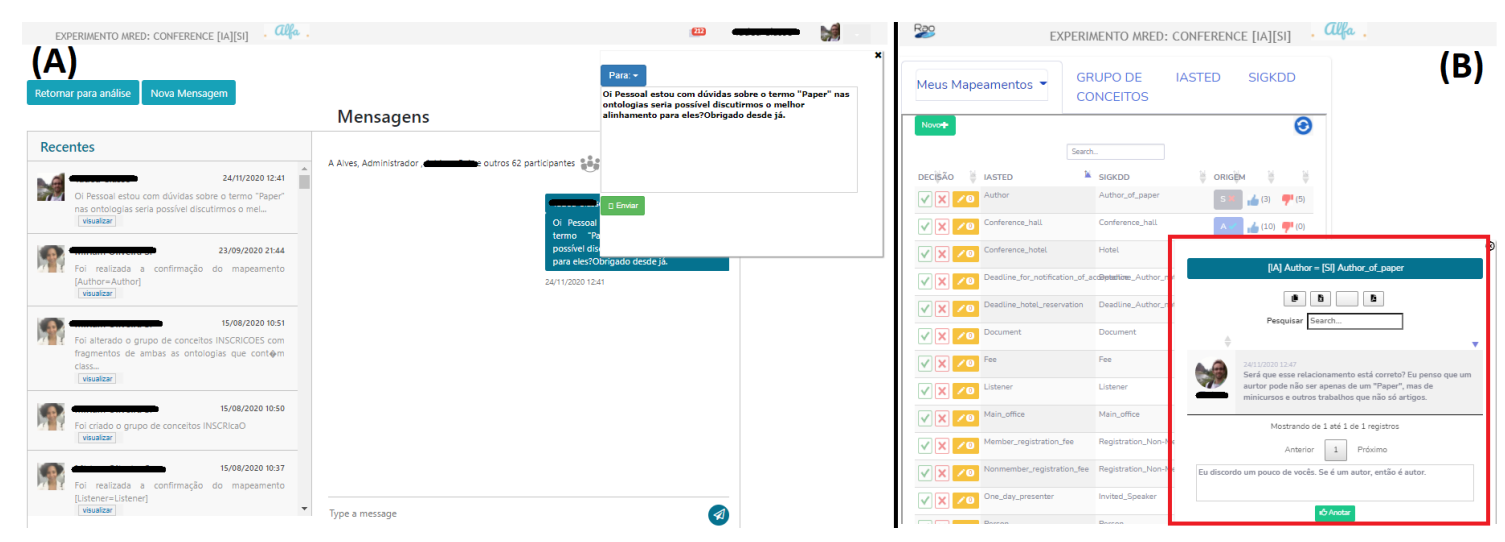

Figura: 2. Dimensão de Comunicação no RAOSystem

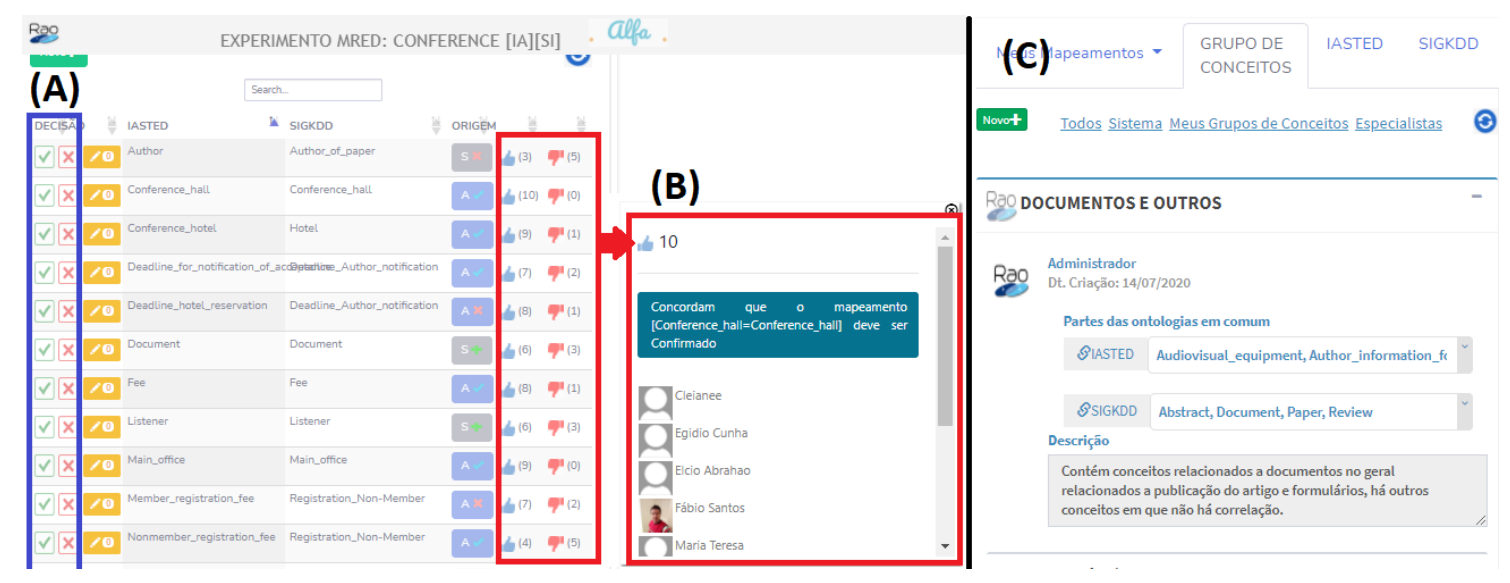

Figura: 3. Dimensão de Cooperação no RAOSystem

\section{Estudo da Colaboração}

\subsection{Planejamento do Estudo}

Nesta pesquisa os aspectos colaborativos do RAOSystem foram analisados por meio de um estudo quasi-experimental, os quais fazem parte de uma classe de estudo de natureza empírica, menos controlados que experimentos clássicos [Campbell e Stanley 2015].

Os objetivos dos estudos são apresentados através da abordagem GQM (GoalQuestion-Metric [Basili 1992] como: analisar o RAOSystem; com o propósito de verificação; no que diz respeito ao uso dos aspectos colaborativos implementados no software; na perspectiva dos especialistas de domínio; no contexto de reparação de alinhamento de ontologias. 


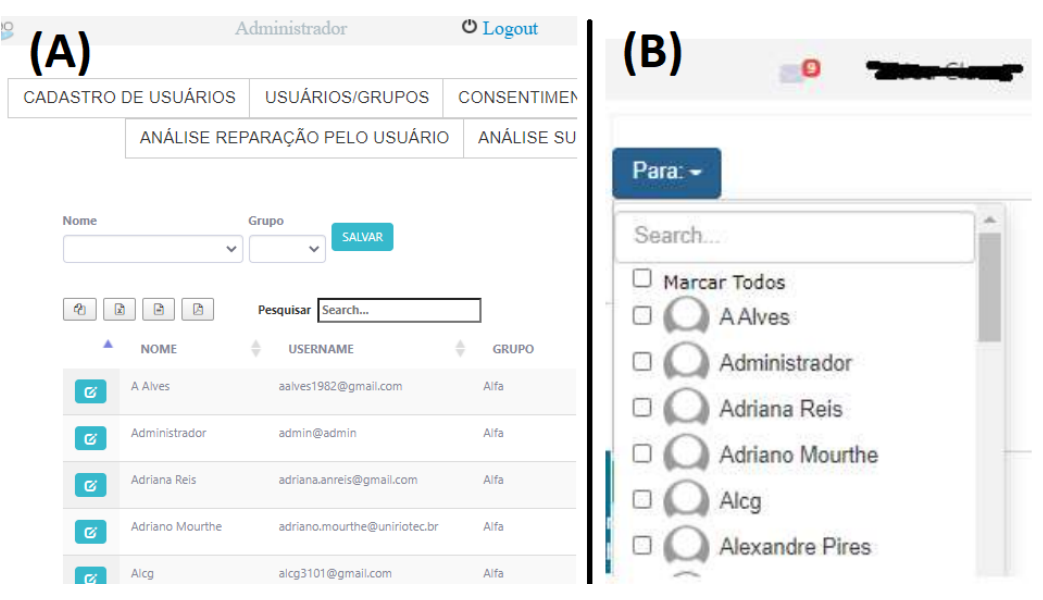

Figura: 4. Dimensão de Coordenação no RAOSystem

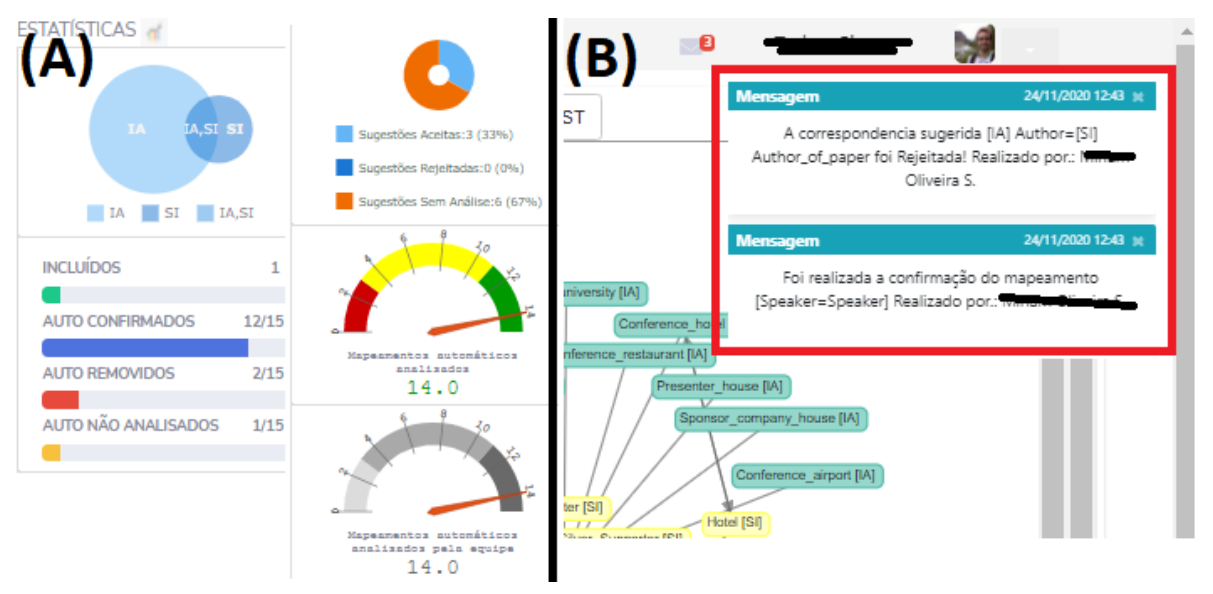

Figura: 5. Dimensão de Percepção no RAOSystem

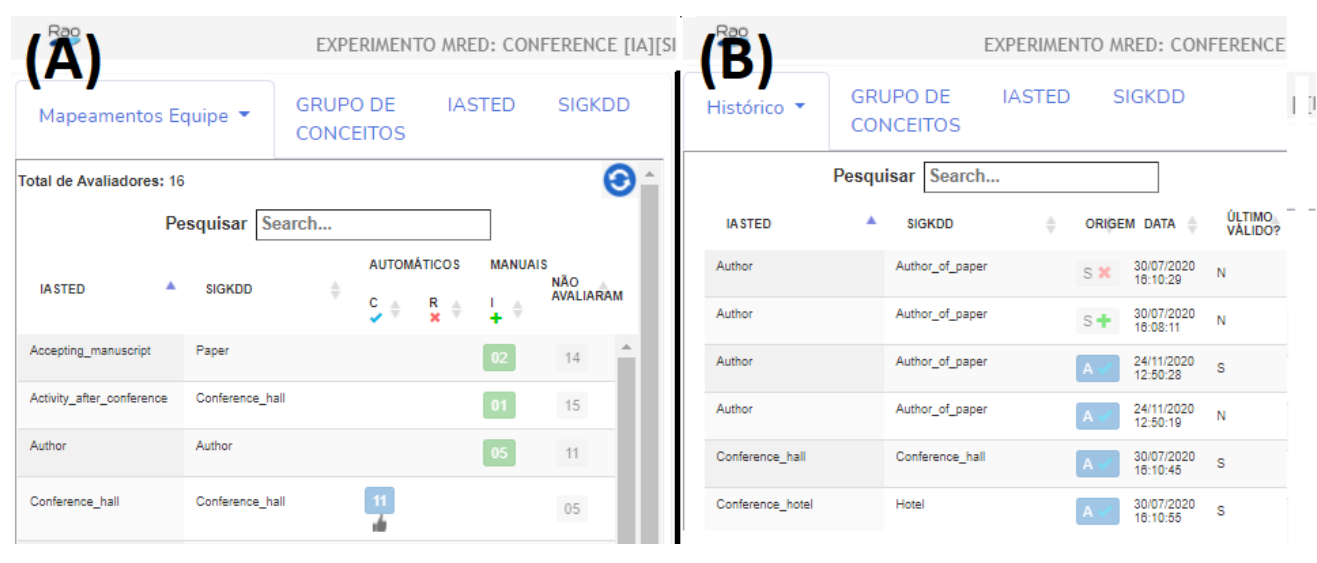

Figura: 6. Dimensão de Memória no RAOSystem

Os participantes da pesquisa foram selecionados por conveniência, incluindo estudantes, professores e pesquisadores devido a serem "especialistas de domínio" sobre conferências, simpósios e workshops. No estudo, ontologias deste domínio foram usadas no RAOSystem. Assim o estudo foi projetado em 3 etapas: 1) instrução: uso de vídeos e tutoriais de apresentação; 2) treinamento: uso do sistema para a reparação guiada 
de um alinhamento simples; 3) execução: reparação de alinhamento de ontologias de conferência.

Para coleta de dados, o RAOSystem implementou a captura de log dos cliques de mouse dos participantes, obtendo informações se a funcionalidade foi usada, por exemplo. Com estes dados foi possível calcular o uso dos recursos colaborativos pelos participantes, considerando os recursos descritos na Tabela 1.

Tabela: 1. Indicadores de trabalho colaborativo

\begin{tabular}{|l|l|}
\hline TC1 & Utilização do recurso colaborativo anotação \\
\hline TC2 & Utilização do recurso colaborativo "concordam” com o alinhamento do termo \\
\hline TC3 & Utilização do recurso colaborativo discordam com o alinhamento do termo \\
\hline TC4 & Utilização do recurso colaborativo mapeamento equipe \\
\hline TC5 & Utilização do recurso colaborativo mensagem \\
\hline
\end{tabular}

Desta maneira o trabalho colaborativo foi calculado de maneira simples, apenas considerando a contagem se a funcionalidade foi usada ou não pelo participante (TC $=T C 1+T C 2+T C 3+T C 4+T C 5)$. Além dos dados coletados pelo próprio sistema, os participantes foram convidados a responder um questionário (Tabela 2) final para a coleta das impressões ${ }^{4}$. O questionário considerou uma escala Likert variando de 1 (discordo totalmente) a 5 (concordo totalmente).

Tabela: 2. Itens do questionário de opinião

\begin{tabular}{|l|l|}
\hline Q01 & É fácil lembrar como enviar uma mensagem para outros especialistas no RAO \\
\hline Q02 & A utilização da ferramenta RAO possibilita trabalho em equipe \\
\hline Q03 & $\begin{array}{l}\text { As formas de comunicação disponibilizadas pela ferramenta RAO para interação entre os participantes (mensagens, anotações) } \\
\text { permite prender com conteúdo colocados por outros participantes }\end{array}$ \\
\hline Q04 & $\begin{array}{l}\text { As formas de comunicação disponibilizadas pela ferramenta RAO entre os participantes (mensagens, anotações e escolhas feitas por } \\
\text { outros usuários) permite tomar decisões (incluir, remover, confirmar mapeamentos) embasadas nestes conteúdos }\end{array}$ \\
\hline Q05 & $\begin{array}{l}\text { A ferramenta RAO proporciona recursos para execução de trabalho colaborativo sem depender da presença física dos outros } \\
\text { participantes }\end{array}$ \\
\hline Q06 & $\begin{array}{l}\text { A ferramenta RAO proporciona recursos para execução de trabalho colaborativo sem depender que outros participantes estejam } \\
\text { logados simultaneamente }\end{array}$ \\
\hline Q07 & No geral, acho a ferramenta RAO útil para executar a Tarefa de Reparação \\
\hline Q08 & $\begin{array}{l}\text { É importante consultar a opinião de outros especialistas de domínio através da troca de mensagens e anotações durante o processo } \\
\text { de RAO. }\end{array}$ \\
\hline Q09 & Você consultou a troca de mensagens e anotações durante o processo de RAO. \\
\hline
\end{tabular}

A execução do estudo contou com 10 participantes voluntários, participando de forma online. A coleta dos dados aconteceu de forma individual e independente. Todos os dados foram analisados, tratados e sumarizados nas Planilhas do Google.

As principais ameaças consideradas no estudo foram: 1) desgaste: tempo de execução do estudo, porém como tratamento, o participante poderia pausar o trabalho e retomar em outro momento; 2) falta de treinamento: mas para minimizar, foram feitos vídeos, tutoriais e uma seção de treino; 3) expectativa do pesquisador: o pesquisador não teve contato com os participantes e; 4) generalização: foi usado apenas um domínio de ontologias, limitando o escopo do estudo.

${ }^{4}$ Dados do estudo: https: / / bit.1y/SBSC2021_RAO 


\subsection{Resultados do Estudo}

Com a finalização da execução do estudo os dados foram coletados e sumarizados em tabelas. A Tabela 3 apresenta os dados relacionados aos aspectos colaborativos implementados e analisados no estudo. É possível perceber que alguns recursos de colaboração foram usados mais que outros, como exemplo: o uso das anotações (TC1), no qual grande parte dos participantes a usaram (70\%); uso de concordância (TC2) e discordância (TC3) em termos do alinhamento (60\%). Também foi possível perceber uma subutilização da visualização do mapeamento em equipe (TC4) (30\%), tendo poucos participantes o usando.

Tabela: 3. Contagem de uso de aspectos colaborativos pelos participantes

\begin{tabular}{|c|c|c|c|c|c|c|c|c|c|c|c|c|}
\hline & P1 & P2 & P3 & P4 & P5 & P6 & P7 & P8 & P9 & P10 & Uso TC & Média \\
\hline TC1 & 1 & 1 & 1 & 1 & 1 & 0 & 1 & 1 & 0 & 0 & 7 & 0,7 \\
\hline TC2 & 0 & 1 & 1 & 1 & 1 & 0 & 1 & 1 & 0 & 0 & 6 & 0,6 \\
\hline TC3 & 0 & 0 & 1 & 1 & 1 & 0 & 1 & 0 & 1 & 1 & 6 & 0,6 \\
\hline TC4 & 0 & 1 & 0 & 0 & 0 & 1 & 1 & 0 & 0 & 0 & 3 & 0,3 \\
\hline TC5 & 1 & 1 & 0 & 1 & 0 & 0 & 0 & 0 & 0 & 1 & 4 & 0,4 \\
\hline TC Participante & 2 & 4 & 3 & 4 & 3 & 1 & 4 & 2 & 1 & 2 & & \\
\hline Média & 0,4 & 0,8 & 0,6 & 0,8 & 0,6 & 0,2 & 0,8 & 0,4 & 0,2 & 0,4 & & \\
\hline
\end{tabular}

O uso dos recursos colaborativos pelos participantes também pode ser observado tanto na Tabela 3, quanto na Figura 7. Nela é possível observar que nenhum participante usou os 5 recursos colaborativos disponibilizados. Contudo, os participantes 2, 4 e 7, utilizaram $80 \%$ dos recursos disponíveis. Destes participantes, os recursos mais usados foram as anotações e a concordância com anotações. Por outro lado, os participantes 6 e 9, utilizaram apenas $20 \%$ das funcionalidades. Sendo que o participante 6 apenas usou o recurso de visualização do mapeamento da equipe, enquanto o participante 9 , usou a discordância.

Figura: 7. Recursos de colaboração usados pelos participantes

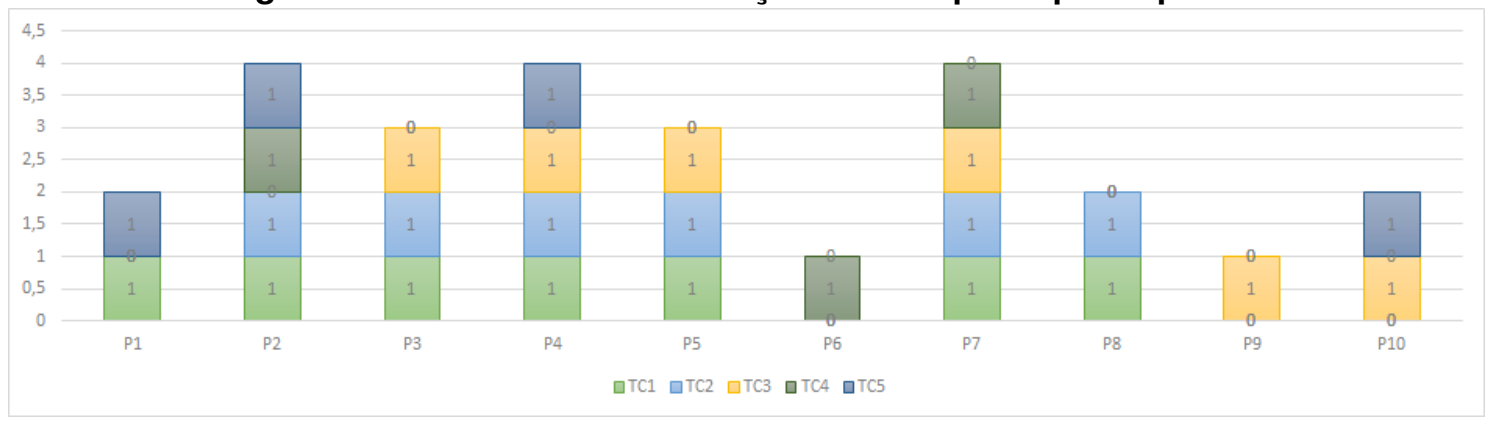

As respostas dos participantes são mostrados na Tabela 4. De forma geral, os participantes concordaram com as questões apresentadas, exceto em (Q09 - média = 2,7), a qual teve um reação neutra, embora os dados dos cliques sobre os recursos colaborativos, mostrados acima, indicaram que elas foram usadas pelos participantes. Isso indica que os participantes concordaram que os recursos colaborativos implementados contribuíram com a tarefa de RAO usando o RAOSystem.

Assim, entende-se que a partir desta análise, os recursos colaborativos podem contribuir com o processo de RAO. Os resultados apontaram que recursos associados a 
Tabela: 4. Respostas do questionário de opinião

\begin{tabular}{|r|c|c|c|c|c|c|c|c|c|}
\hline & Q01 & Q02 & Q03 & Q04 & Q05 & Q06 & Q07 & Q08 & Q09 \\
\hline P1 & 4 & 3 & 3 & 4 & 4 & 4 & 4 & 5 & 1 \\
\hline P2 & 5 & 5 & 5 & 5 & 5 & 5 & 5 & 5 & 5 \\
\hline P3 & 5 & 5 & 4 & 5 & 5 & 4 & 5 & 3 & 1 \\
\hline P5 & 5 & 5 & 5 & 4 & 5 & 5 & 5 & 3 & 2 \\
\hline P6 & 3 & 4 & 4 & 4 & 4 & 4 & 4 & 4 & 1 \\
\hline P7 & 4 & 3 & 5 & 5 & 5 & 5 & 5 & 4 & 1 \\
\hline P8 & 5 & 5 & 5 & 5 & 5 & 5 & 5 & 5 & 3 \\
\hline P9 & 5 & 5 & 5 & 2 & 3 & 4 & 4 & 5 & 3 \\
\hline P10 & 5 & 5 & 5 & 5 & 5 & 5 & 5 & 5 & 5 \\
\hline P11 & 4 & 4 & 5 & 5 & 4 & 4 & 4 & 5 & 5 \\
\hline Média & $\mathbf{4 , 5}$ & $\mathbf{4 , 4}$ & $\mathbf{4 , 6}$ & $\mathbf{4 , 4}$ & $\mathbf{4 , 5}$ & $\mathbf{4 , 5}$ & $\mathbf{4 , 6}$ & $\mathbf{4 , 4}$ & $\mathbf{2 , 7}$ \\
\hline Desvio Padrão & $\mathbf{0 , 7}$ & $\mathbf{0 , 8}$ & $\mathbf{0 , 7}$ & $\mathbf{1 , 0}$ & $\mathbf{0 , 7}$ & $\mathbf{0 , 5}$ & $\mathbf{0 , 5}$ & $\mathbf{0 , 8}$ & $\mathbf{1 , 8}$ \\
\hline & & & & & & &
\end{tabular}

cooperação (TC1, TC2 e TC3), junto com recursos de comunicação (TC1 e TC5), podem contribuir com a melhora da qualidade de ontologias no processo de RAO. Esses recursos apoiaram o trabalho colaborativo na ferramenta RAOSystem, no qual os especialistas de domínio puderam contribuir uns com os outros sobre a ontologia de conferências.

\section{Considerações Finais}

A interoperabilidade entre sistemas que usam ontologias como base de compartilhamento de conhecimento depende de um processo conhecido por alinhamento de ontologias. Porém nem sempre os alinhamentos obtidos por meio de meios automatizados apresentam a união das ontologias de forma eficiente. Para verificar, confirmar e corrigir alinhamentos entre ontologias, os especialistas de domínio precisam realizar o processo manual de reparação de alinhamento de ontologias. No processo de reparação, a colaboração entre os especialistas de domínio desempenha um papel fundamental. O conhecimento de vários especialistas, confirmando, trocando mensagens e opiniões, contribui com o conhecimento coletivo e, consequentemente, com reparações de ontologias melhores. Este trabalho apresentou o sistema RAOSystem, responsável por dar suporte aos especialista de domínio na tarefa de RAO. Esse sistema, conta com várias funcionalidades colaborativas, inspiradas no modelo de colaboração 3C. Neste sentido, este trabalho verificou esses recursos colaborativos através de um estudo quasi-experimental usando análise mista de dados (qualitativa e quantitativa) para analisar os dados.

A análise dos dados indicou que os recursos colaborativos foram usados pelos participantes dos estudos. Uns mais que os outros, sendo possível destacar as anotações (comentários) nos alinhamentos e a confirmação ou rejeição dos alinhamentos dos termos. Outros foram usados menos como é o exemplo do mapeamento da equipe, representando a memória coletiva. De maneira similar, alguns participantes colaboraram, ou usaram mais recursos colaborativos que outros. Aos responderem um questionário de opinião ao final do estudo, praticamente a totalidade dos participantes indicaram que o trabalho colaborativo é importante no processo de RAO. Contudo, eles indicaram um comportamento de neutralidade quando questionados se eles as usaram. Confirmando os dados dos cliques que indicaram o uso por poucos participantes.

Este trabalho, embora finalizado, apresenta um dos ciclos de pesquisa. Ele faz parte de um trabalho maior sobre a definição de um guia/modelo para a criação de sistemas que apoiem especialistas de domínio no processo de RAO. A verificação dos aspectos colaborativos foi apenas um dos itens do trabalho. Porém com os resultados observados 
aqui, nesta parte da pesquisa, é possível dizer que o trabalho colaborativo é importante nessa tarefa. O próximo passo da pesquisa é verificar se o trabalho colaborativo influencia na qualidade do alinhamento de ontologias, permitindo uma maior precisão e cobertura de entre os termos semanticamente similares, e, consequentemente, a interoperabilidade de dados entre sistemas heterogêneos.

\section{Referências}

Banouar, O. e Raghay, S. (2016). Interoperability of information systems through ontologies: State of art. International Journal of Computer Science and Information Security, 14(8):392.

Basili, V. R. (1992). Software modeling and measurement: the goal/question/metric paradigm. Technical report.

Borges, M. R. d. S. (2011). Conhecimento coletivo. In Fuks, H. e Pimentel, M., editors, Sistemas Colaborativos, chapter 12, pages 186-205. Elsevier Brasil, Rio de Janeiro.

Brodaric, B. e Gahegan, M. (2010). Ontology use for semantic e-science. Semantic Web, 1(1, 2):149-153.

Campbell, D. T. e Stanley, J. C. (2015). Experimental and quasi-experimental designs for research. Ravenio Books.

Euzenat, J. (2014). First experiments in cultural alignment repair (extended version). In European Semantic Web Conference, pages 115-130. Springer.

Euzenat, J. e Rousset, M.-C. (2020). Semantic web. In A Guided Tour of Artificial Intelligence Research, pages 181-207. Springer.

Euzenat, J., Shvaiko, P., et al. (2007). Ontology matching, volume 18. Springer.

Falconer, S. M., Noy, N. F., e Storey, M.-A. D. (2007). Ontology mapping-a user survey. In $O M$. Citeseer.

Faria, D., Pesquita, C., Santos, E., Palmonari, M., Cruz, I. F., e Couto, F. M. (2013). The agreement makerlight ontology matching system. In OTM Confederated International Conferences"On the Move to Meaningful Internet Systems , pages 527-541. Springer.

Fuks, H., Raposo, A., Gerosa, M. A., et al. (2008). The 3c collaboration model. In Encyclopedia of E-collaboration, pages 637-644. IGI Global.

Gargouri, F. e Jaziri, W. (2010). Ontology theory, management, and design: Advanced tools and models. Information Science Reference.

Harrow, I., Balakrishnan, R., Jimenez-Ruiz, E., Jupp, S., Lomax, J., Reed, J., Romacker, M., Senger, C., Splendiani, A., Wilson, J., e Woollard, P. (2019). Ontology mapping for semantically enabled applications. Drug discovery today, 24:2068-2075.

Karampatakis, S., Bratsas, C., Zamazal, O., Filippidis, P. M., e Antoniou, I. (2018). Alignment: a hybrid, interactive and collaborative ontology and entity matching service. Information, 9(11):281.

Li, H., Dragisic, Z., Faria, D., Ivanova, V., Jiménez-Ruiz, E., Lambrix, P., e Pesquita, C. (2019). User validation in ontology alignment: functional assessment and impact. The Knowledge Engineering Review, 34. 
Li, Y., Stroe, C., e Cruz, I. F. (2015). Interactive visualization of large ontology matching results. InVOILA@ISWC, page 37.

Massmann, S., Raunich, S., Aumüller, D., Arnold, P., e Rahm, E. (2011). Evolution of the coma match system. In Proceedings of the 6th International Conference on Ontology Matching-Volume 814, pages 49-60. CEUR-WS. org.

Meilicke, C. (2011). Alignment incoherence in ontology matching. $\mathrm{PhD}$ thesis, Universität Mannheim.

Meilicke, C., Stuckenschmidt, H., e Tamilin, A. (2008). Supporting manual mapping revision using logical reasoning. In AAAI, pages 1213-1218.

Meilicke, C., Stuckenschmidt, H., e Tamilin, A. (2009). Reasoning support for mapping revision. Journal of logic and computation, 19(5):807-829.

Meng, Z., Brooke, J., e Sakellariou, R. (2016). Semantic accountable matchmaking for e-science resource sharing. In 2016 IEEE 12th International Conference on e-Science (e-Science), pages 282-286. IEEE.

Mistrík, I., Grundy, J., Van der Hoek, A., e Whitehead, J. (2010). Collaborative software engineering: challenges and prospects. In Collaborative software engineering, pages 389-403. Springer.

Pesquita, C., Faria, D., Santos, E., e Couto, F. M. (2013). To repair or not to repair: reconciling correctness and coherence in ontology reference alignments. In Proc. 8th ISWC ontology matching workshop (OM), Sydney (AU), page this volume.

Pimentel, M. e Fuks, H. (2012). Sistemas colaborativos. Elsevier.

Roseman, M. e Greenberg, S. (1995). Groupkit a groupware toolkit for building realtime conferencing applications. In Readings in Human-Computer Interaction, pages 390-397. Elsevier.

Santos, M. O., de Mello, C. E. R., e Classe, T. M. (2020). A useful tool to support the ontology alignment repair. In Brazilian Conference on Intelligent Systems, pages 201-215. Springer.

Santos, M. O. d. (2020). Modelo para Avaliação de Usabilidade de Usuários em Ferramentas de Visualização para Reparação de Alinhamento de Ontologias: Uma Metodologia a partir de Dados Analíticos. PhD thesis, Programa de Pós-Graduação em Informática. Universidade Federal do Estado do Rio de Janeiro, Rio de Janeiro.

Silva, J., Baião da, F. A., e Revoredo, K. (2016). Alinhamento interativo de ontologias usando anti-padrões de alinhamento: Um primeiro experimento. In Anais Principais do XII Simpósio Brasileiro de Sistemas de Informação, pages 208-215. SBC.

Smirnov, A. e Shilov, N. (2013). Ontology matching in collaborative recommendation system for plm. International Journal of Product Lifecycle Management, 6(4):322338.

Xue, X. e Liu, J. (2017). Collaborative ontology matching based on compact interactive evolutionary algorithm. Knowledge-Based Systems, 137:94-103. 